\title{
Délétion de gènes de la région constante des chaînes lourdes des immunoglobulines humaines
}

Marie-Paule Lefranc Gérard Lefranc Professeurs à l'Université de Montpellier-II

\section{REFERENCES}

I. Natvig JB, Kunkel HG. Human immunoglobulins: classes, subclasses, genetic variants and idiotypes. Adv Immunol 1973; 16: 1-59.

2. W. H. O. Revicw of the notation for the allotypic and related markers of human immunoglobulins. W. H. O. mecting on human immunoglobulin allotypic markers. 7 Immunogenetics $1976 ; 3$ : 357-62.

3. Loghem E van, Aalberse RC, Matsumoto H. A genctic marker of human IgE heavy chains, Em(I). Vox Sang 1984; 46: 195-206.

\section{ADRESSE}

M.-P. Lefranc, G. Lefranc : laboratoire d'immunogénétique, U.A. Cnrs i rq I génétique rioléculaire, université des sciences et techniques du Languedoc, Place E. Bataillon, 3406o Montpellier
Des délétions considérables des gènes codant pour les régions constantes des chaînes lourdes d'immunoglobuline sont observées chez des sujets normaux. Probablement secondaires à des phénomènes de recombinaison inégale dans une famille multigénique, ces délétions témoignent de la flexibilité évolutive de ce système génétique.

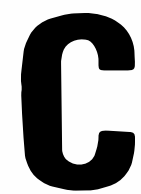
hez l'homme, il existe 5 classes d'immunoglobulines (IgM, IgD, IgG, IgE et $\operatorname{Ig} A$ ) définies par les déterminants antigéniques isotypiques, les propriétés physicochimiques et les activités biologiques de leurs chaînes lourdes $(\mu, \delta$, $\gamma$, $\varepsilon$ et $\alpha$, respectivement). De plus, des sous-classes ont été décrites pour les $\operatorname{IgG}\left(\operatorname{IgG} I, \operatorname{IgG}_{2}, \operatorname{IgG}_{3}\right.$ et $\left.\mathrm{IgG}_{4}\right)$ et les $\operatorname{IgA}\left(\operatorname{Ig} \mathrm{A}_{1}\right.$ et $\left.\operatorname{Ig} \mathrm{A}_{2}\right)$ [I]. Les régions constantes $(\mathrm{CH})$ des chaînes lourdes $\gamma_{1}, \gamma_{2}, \gamma_{3}, \alpha_{2}$ et $\varepsilon$ peuvent aussi être identifiées par des déterminants antigéniques allotypiques *, véritables marqueurs des $\operatorname{Ig} G_{1}, \operatorname{Ig} G_{2}, \operatorname{Ig} G_{3}, \operatorname{Ig} A_{2}$ et $\mathrm{IgE}$, dénommés pour cette raison allotypes Gim, G2m, G3m, A2m et $\operatorname{Em}[2,3]$. Ils représentent également de précieux marqueurs utilisés pour caractériser les populations

\footnotetext{
* Allotypes, déterminants antigéniques allot ypiques = déterminants codés par des allèles, c'est-à-dire par des variants d'un même gène. Tous les allèles correspondent à un même "locus "génétique.
}

humaines $[4,5]$ et pour la génétique des immunoglobulines [6-9]. En particulier, la transmission de groupes inhabituels d'allotypes permet de révéler des événements génétiques (mutations ponctuelles, duplications, délétions, échanges d'exons, conversions géniques, recombinaisons inégales intragéniques à l'origine de gènes hybrides...) qui se sont produits au niveau des régions codantes [6-9]. Grâce aux sondes moléculaires spécifiques, ces événements génétiques qui affectent les gènes $\mathrm{CH}$ des immunoglobulines humaines (localisés sur le chromosome I4 [Io] au niveau de la bande 932 [II]) sont maintenant étudiés au niveau de l'ADN. Récemment, des délétions importantes englobant plusieurs gènes $\mathrm{CH}$ ont été décrites.

\section{Gènes $\mathrm{CH}$ et sondes spécifiques}

Gènes $\mathrm{C} \mu$ et $\mathrm{C} \delta$. Lorsque l'ADN humain est digéré avec une enzyme de restriction telle que BamHI et 
hybridé, selon la méthode de Southern, avec une sonde $\mathrm{C} \mu$ [12], on observe un unique fragment de 17 kilobases $(\mathrm{kb})$ correspondant à ce gène, tandis qu'une sonde $C \delta$ révèle un fragment de I i kb [13].

Gènes $\mathbf{C} \gamma$. Une sonde $\mathrm{C} \gamma_{3}$ ou $\mathrm{C} \gamma_{4}$ [14] révèle tous les gènes $C \gamma$ par suite de l'homologie très importante, de l'ordre de $95 \%$ [ I 5], entre les différents gènes $\mathrm{C} \gamma$ humains. Par ailleurs, le nombre des fragments détectés est variable (de 5 à 8 si l'ADN est digéré par BamHI), d'un groupe d'individus à un autre, par suite de l'existence d'un polymorphisme de longueur des fragments de restriction [I3] ( figure $I-N)$.

Gènes $\mathrm{C} \varepsilon$. Une sonde $\mathrm{C} \varepsilon[16]$ révèle trois fragments $\mathrm{BamHI}$ de $2,7 \mathrm{~kb}, 6 \mathrm{~kb}$ et $9 \mathrm{~kb}$ [16-18] ( $f i-$ gure $2-N)$. Le fragment de $2,7 \mathrm{~kb}$ correspond au gène $C \varepsilon$ actif [ $16-18$ ] tandis que les deux autres de 6 et $9 \mathrm{~kb}$ représentent des pseudogènes appelés respectivement $\psi \varepsilon_{1}$ et $\psi \varepsilon 2$ $[17,19]$. Le pseudogène $\psi \varepsilon$ I est situé sur le chromosome I 4 entre les gènes $C \gamma_{I}$ et $C \alpha_{I}$ [I9], le pseudogène $\psi \varepsilon 2$ est un gène ayant perdu ses introns et localisé sur le chromosome 9 [20].

Gènes $\mathbf{C} \alpha$. Pour étudier les gènes $\mathrm{C} \alpha$, l'ADN est coupé par une autre enzyme de restriction PstI. Une sonde $\mathrm{C} \alpha$ [19] révèle un fragment de I, $2 \mathrm{~kb}$ pour $\alpha$ I et un autre de $2 \mathrm{~kb}$ pour $\alpha_{2}[\mathrm{I} 3, \mathrm{I} 9](\mathrm{fig} .3-N)$.

\section{Délétion des gènes $C \gamma 1$, $C_{\gamma 2}, C_{\gamma 4}, \psi \gamma, \psi \varepsilon 1, C_{\alpha 1}$}

La présence des seuls allotypes des $\mathrm{IgG}_{3}$ et l'absence des autres marqueurs allotypiques ainsi que celle des déterminants isotypiques des autres sous-classes d'IgG et des $\operatorname{Ig} A_{I}$ ont permis de déceler une absence simultanée des IgG I, IgG2, $\mathrm{IgG}_{4}$ et Ig AI chez une Tunisienne de 75 ans, en bonne santé (désignée $\mathrm{TAK}_{3}$, famille Hass) [2I]. Seules les classes et sous-classes IgM, IgD, $\operatorname{Ig} G_{3}, \operatorname{Ig} E$ et $\operatorname{Ig} A_{2}$ étaient présentes.

L'ADN a été préparé à partir des leucocytes du sang, digéré par BamHI (ou Pst I comme indiqué plus haut) et hybridé avec les sondes $\mathrm{C} \mu, \mathrm{C} \delta, \mathrm{C} \gamma, \mathrm{C} \varepsilon$ et $\mathrm{C} \alpha$. Ces hybridations ont révélé la présence des seuls gènes $\mathrm{C} \mu, \mathrm{C} \delta, \mathrm{C} \gamma_{3}, \mathrm{C} \varepsilon$ et $\mathrm{C} \alpha_{2}$ et,

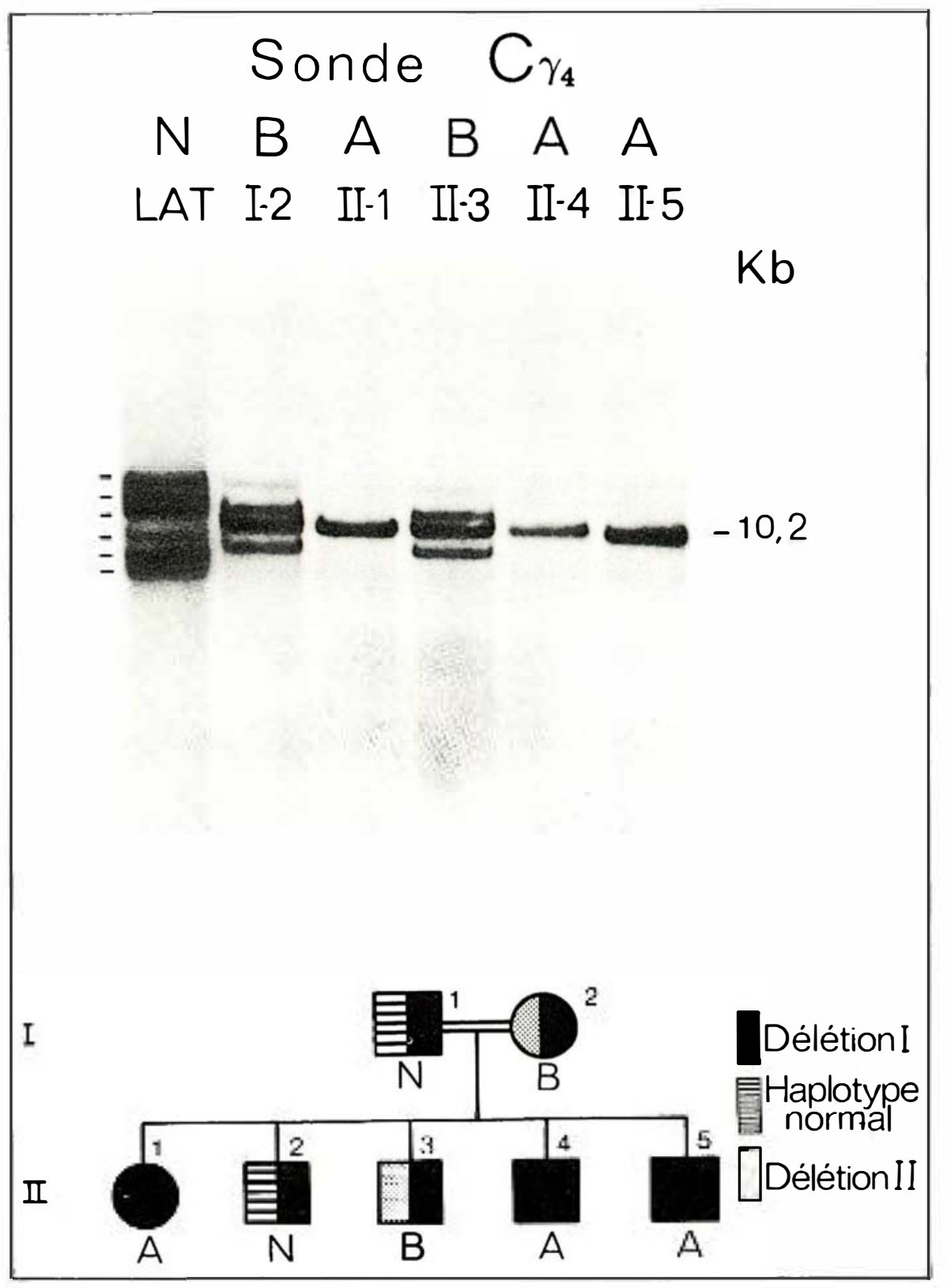

Figure 1. Hybridation, avec une sonde $C \gamma$, des ADN génomiques préalablement digérés par Bam HI [22].

$N$ : ADN génomique normal

A: ADN des membres de la famille Tou, homozygotes pour la délétion I

$B$ : ADN des membres de la famille Tou, présentant la délétion / sur un chromosome 14 et la délétion I/ sur l'autre chromosome 14. 


\section{REFERENCES}

4. Steinberg AG. Contribution of the $\mathrm{Gm}$ and Inv allotypes to the characterization of human populations. Isr 7 Med Sci 1973; 9: 1249-56.

5. Lefranc $G$, de Lange $G$, Rivat $L$ et al. Gm, $\mathrm{Am}$ and $\mathrm{Km}$ immunoglobulin allotypes of two populations in Tunisia. Hum Genet 1979; 50: 199-2 I I

6. Lefranc G, Dumitresco SM, Salier JP et al. Familial lack of the $\mathrm{IgG}_{3}$ subclass: gene elimination or turning off expression and neutral evolution in the immune system. 7 Immunogenet 1979; 6: 2 I 5-2 I

7. Loghem E, Sukernik RI, Osipova LP et al. Gene deletion and gene duplication within the cluster of human heavy chain genes. Selective absence of IgG subclasses. I Immunogenet 1980; 7: 285-99.

8. Lefranc G, Lef ranc MP, Helal AN et al. Unusual heavy chains of human IgG immunoglobulins: rearrangements of the $\mathrm{CH}$ domain exons. 7 Immunogenet 1982; 9: 1-9.

9. Lef ranc MP, Helal AN, de Lange G, Chaabani $H$, van Loghem $E$, Lef ranc $G$. Gene conversion in human immunoglobulin locus shown by unsual location of IgG allotypes. FEBS Letters 1986 ; 196: 96-1 02

10. Croce CM, Shander M, Martinis J et al. Chromosomal location of the genes for human immunoglobulin heavy chains. Proc Natl Acad Sci USA 1979; 76: 3416-9.

I I. Kirsch IR, Morton CC, Nakahara K, Leder $P$. Human immunoglobulin heavy chain genes map to a region of translocations in malignant $B$ lymphocytes. Science 1982; $216: 301-3$.

12. Rabbitts TH, Forster A, Milstein CP. Human immunoglobulin heavy chain genes: Evolutionary comparisons of $\mathrm{C} \mu, \mathrm{C} \delta$ and $\mathrm{C} \gamma$ genes and associated switch sequences. Nucleic Acids Res I981; 9: 4509-24

13. Lefranc MP, Lefranc G, Rabbitts TH. Inherited deletion of immunoglobulin heavy chain constant region genes in normal human individuals. Nature 1982; 300: 760-2.

14. Krawinkel U, Rabbitts TH. Comparison of the hinge-coding segments in human immunoglobulin gamma heavy chain genes and the linkage of the gamma 2 and gamma 4 subclass genes. $E M B O F$ 1982; 1: 403-7.

15. Huck S, Fort $P$, Crawford D, Lefranc MP, Lef ranc $G$. Sequence of a human immunoglobulin $\gamma_{3}$ heavy chain constant region gene. Comparison with the other $\mathrm{C} y$ human genes.' Nucleic Acids Res 1986; 14: 1779-89.

16. Flanagan JG, Rabbitts TH. The sequence of human immunoglobulin epsilon heavy chain constant region gene, and evidence for three non-allclic genes. $E M B O{ }_{1}$ 1982; 1: 655-bo.

17. Max EE, Battey J, Ney R, Kirsch IR, Leder $P$. Duplication and deletion in the human immunoglobulin $\varepsilon$ genes. Cell 1982; 29: 69I-9.

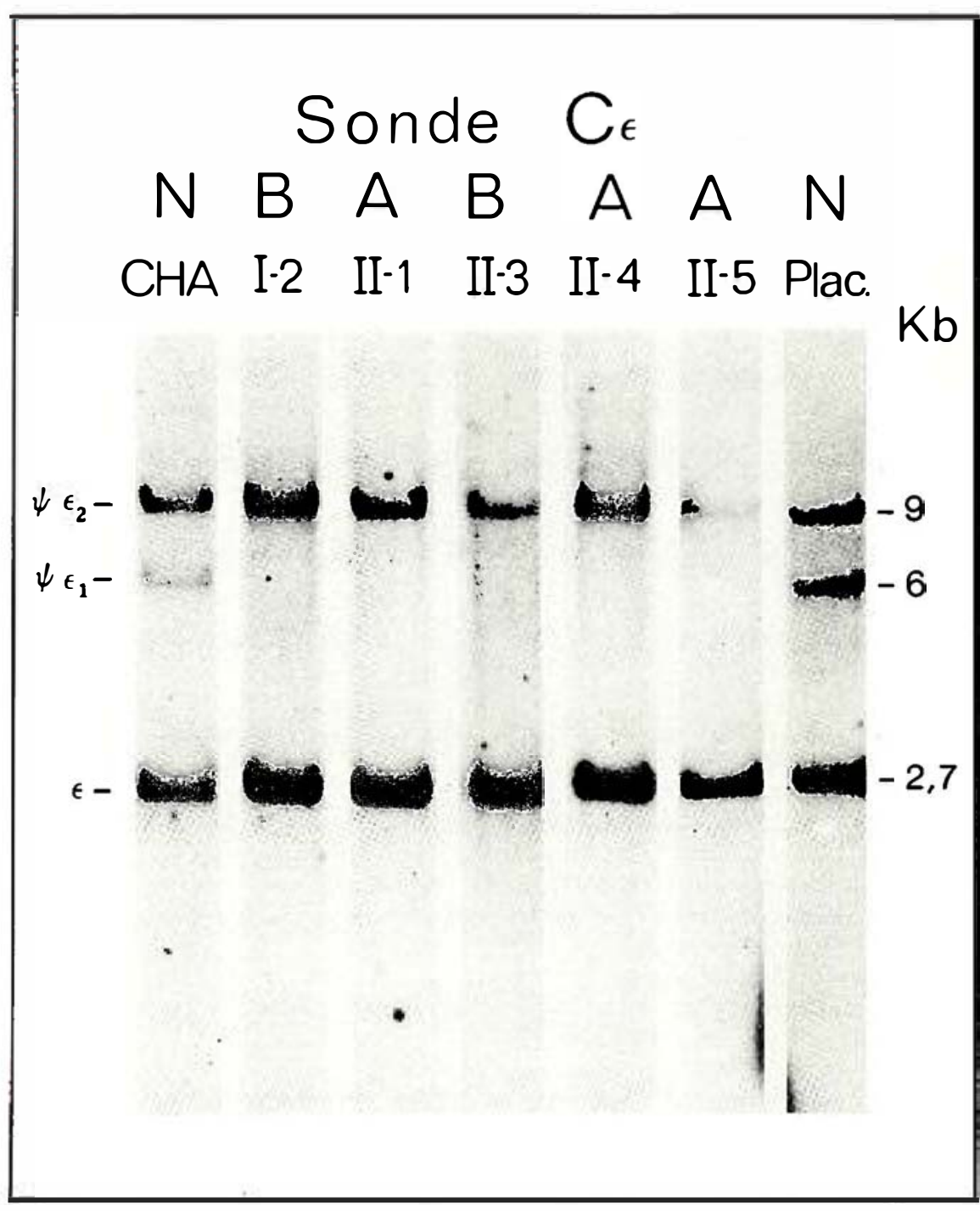

Figure 2. Hybridation, avec une sonde $C_{\varepsilon_{0}}$ des $A D N$ génomiques préalablement digérés par Bam HI [22]. Pour la signification de N, A, B, se reporter à la légende de la figure 1.

par conséquent, démontré l'absence des gènes $\mathrm{C} \gamma_{\mathrm{I}}, \mathrm{C} \gamma_{2}, \mathrm{C} \gamma_{4}$ et $\mathrm{C} \alpha \mathrm{I}$ ainsi que celle des pseudogènes $\psi \gamma$ et $\psi \varepsilon$ I [ 13$]$. Une délétion semblable a été trouvée chez trois autres personnes appartenant à une seconde famille tunisienne (famille Tou) [22] (figure 1-A) et, plus récemment, chez deux petits-fils de TAK $_{3}$ (M.P.L. et G.L., résultats non publiés). Pour ces six personnes, l'hybridation avec une sonde C $\gamma$ montre une seule bande de 10.2 $\mathrm{kb}$ représentant le gène $\mathrm{C} \gamma 3$, les fragments correspondant aux gènes $\mathrm{C} \gamma_{1}, \mathrm{C} \gamma_{2}, \mathrm{C} \gamma_{4}$ et $\psi \gamma \gamma$ étant absents $[13,22]$. La sonde $\mathrm{C} \varepsilon$ révèle les fragments de 2,7 et $9 \mathrm{~kb}$ correspon- dant respectivement aux gènes $\mathrm{C} \varepsilon$ et $\psi \varepsilon 2$; l'absence du fragment de $6 \mathrm{~kb}$ traduit celle du pseudogène $\psi \varepsilon$ I (figure 2- $A$ ). L'ADN digéré par Pst I et hybridé avec la sonde $\mathrm{C} \alpha$ révèle la seule bande de $2 \mathrm{~kb}$ du gène $\mathrm{C} \alpha 2$, celle de $\mathrm{I}, 2 \mathrm{~kb}$ du gène $\mathrm{C} \alpha_{\mathrm{I}}$ étant absente $[\mathrm{I} 3,22]$ ( $f i-$ gure 3-A). Ainsi, une délétion d'une importante région du chromosome 14, comprenant les gènes $\mathrm{C} \gamma_{1}, \mathrm{C} \gamma_{2}, \mathrm{C} \gamma_{4}, \psi \gamma, \psi \varepsilon_{\mathrm{I}}$ et $\mathrm{C} \alpha_{\mathrm{I}}$, existe à l'état homozygote chez ces six personnes en bonne santé. Au delà des implications immunologiques qui seront discutées plus loin, une telle délétion a permis d'établir l'ordre des gènes $\mathrm{CH}$ chez l'homme. 


\section{Ordro des gènes $\mathrm{CH}$ humains}

Alors que chez la souris, l'ordre des gènes $\mathrm{CH}$ est le suivant [23] :

$5^{\prime}-\mu \stackrel{4,5 \mathrm{~kb}}{=} \delta \stackrel{55 \mathrm{~kb}}{=} \gamma_{3} \stackrel{34 \mathrm{~kb}}{=} \gamma_{\mathrm{I}} \stackrel{21 \mathrm{~kb}}{=}$

$\gamma 2 b \stackrel{15 \mathrm{~kb}}{=} \gamma 2 a \stackrel{14,5 k b}{=} \varepsilon \stackrel{12,5 \mathrm{~kb}}{-} \alpha-3^{\prime}$ la présence, chez l'homme, de pseudogènes $(\psi \gamma$ et $\psi \varepsilon \mathrm{I})$ et de deux gènes $\mathrm{C} \alpha$, laissait prévoir une organisation différente. Deux groupes de gènes $\mathrm{CH}$ ont été identifiés $\gamma_{3}$ $\gamma_{\mathrm{I}-\psi} \psi \varepsilon \mathrm{I}-\alpha \mathrm{I}$ (région $A$, figure 4, p. 5I3), d'une part, et $\gamma_{2}-\gamma_{4}-\varepsilon-\alpha_{2}$ (région $B$ ), d'autre part [19]. L'existence de la délétion précédente (délétion I dans la figure 4) a permis de situer la région $A$ en $5^{\prime}$ de la région $\mathrm{B}$, la délétion débutant en aval (en $3^{\prime}$ ) du gène $\mathrm{C} \gamma_{3}$ et se terminant en amont (en $\left.5^{\prime}\right)$ du gène fonctionnel $\mathrm{C} \varepsilon[13$, I9]. De plus, la délétion I englobant également le pseudogène $\psi \gamma$, il devenait possible de localiser celui-ci entre les gènes $\mathrm{C} \alpha$ I et $\mathrm{C} \gamma_{2}$ [22]. Sachant que $\mathrm{C} \mu$ et $\mathrm{C} \delta$, distants l'un de l'autre de $8 \mathrm{~kb}$ [24], sont situés en amont du groupe $\mathrm{A}$, l'ordre des gènes $\mathrm{CH}$ chez l'homme, est le suivant $[13,19,22$, 24] :

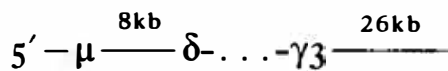

$$
\begin{aligned}
& \gamma_{\mathrm{I}} \stackrel{19 \mathrm{~kb}}{ } \psi \varepsilon \mathrm{I} \stackrel{13 \mathrm{~kb}}{=} \alpha_{\mathrm{I}}-\ldots-\psi \gamma-\ldots \\
& -\gamma_{2} \stackrel{18 \mathrm{~kb}}{=} \gamma_{4} \stackrel{23 \mathrm{~kb}}{=} \varepsilon \frac{10 \mathrm{~kb}}{2} \alpha_{2-3^{\prime}}
\end{aligned}
$$

La distance séparant $\mathrm{C} \delta$ de $\mathrm{C} \gamma_{3}$ n'est pas encore connue avec précision; il en est de même pour la distance entre $\mathrm{C} \alpha_{2}$ et $\mathrm{C} \gamma_{2}$ probablement supérieure à $45 \mathrm{~kb}$ [19].

\section{Délétion}

des gènes $\psi \varepsilon 1-C \alpha 1-\psi \gamma$

Normalement, le nombre et la taille des fragments de restriction de l'ADN des personnes hétérozygotes pour la délétion précédente auraient dû être ceux rencontrés chez toute personne possédant l'ensemble des gènes $\mathrm{CH}$ en raison de la présence supposée d'un chromosome I4 intact. D'une manière surprenante, l'ADN de deux personnes hétérozygotes de la famille Tou a montré l'absence des gènes $\psi \gamma, \psi \varepsilon$ I et $C \alpha$ I [22] (figures $I-B, 2-B, 3-B$ ). Ces $m / s n^{\circ}$ 9, vol. 2, novembre 86 résultats ne pouvaient s'expliquer qu'en admettant l'existence d'une deuxième délétion englobant ces gènes (délétion II dans la figure 4). Deux délétions étaient donc rencontrées dans cette famille: l'une particulièrement importante, puisque s'étendant probablement sur plus de cent kilobases, caractérisée par l'absence des gènes $\gamma_{\mathrm{I}}, \psi \varepsilon_{\mathrm{I}}, \alpha_{\mathrm{I}}$, $\psi \gamma, \gamma_{2}$ et $\gamma_{4}$ (délétion I, figure 4), l'autre plus réduite, bien que de taille encore respectable car supérieure à une cinquantaine de kilobases, couvrant les gènes $\psi \varepsilon_{\mathrm{I}}, \alpha_{\mathrm{I}}$ et $\psi \gamma$ (délétion II, figure 4). Ces personnes hétérozygotes ont une absence sélective en IgAI [22]. Cette observation est intéressante à noter puisque, dans tous les cas d'absence simultanée des deux sous-classes $\operatorname{Ig} \mathrm{A}_{\mathrm{I}}$ et $\operatorname{Ig} \mathrm{A}_{2}$ étudiés par ailleurs, aucune délétion de gènes $C \alpha$ n'a été observée (M.P.L. et T.H. Rabbitts, résultats non publiés).

\section{Autres délétions multigéniques}

Plus récemment, deux autres délétions multigéniques affectant des gènes $\mathrm{CH}$ ont été décrites, également chez des personnes en bonne santé. La première, trouvée à l'état homozygote chez une personne de 5 I ans du Sud de l'Italie, englobe les gènes $\alpha_{1}, \psi \gamma, \gamma_{2}, \gamma_{4}$ et $\varepsilon$ [25]. Cette délétion (délétion III, figure 4) est différente de la délétion I en ce qu'elle n'inclut pas le gène $C_{\gamma_{I}}$ mais, en revanche, comprend le gène $C \varepsilon$ et entraîne par conséquent une absence des IgE. Une personne

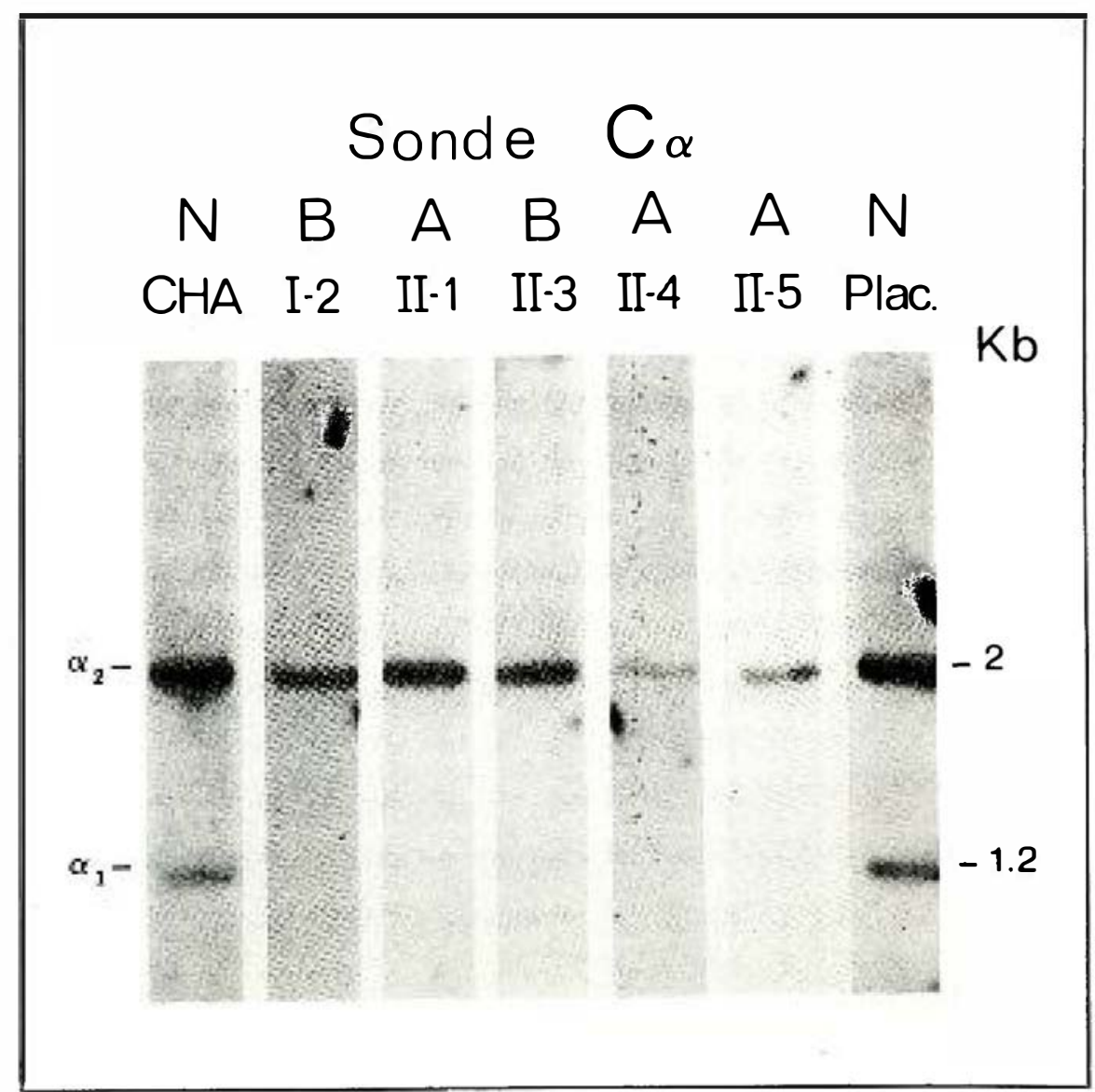

Figure 3. Hybridation, avec une sonde $C \alpha$ des $A D N$ génomiques préalablement digérés par Pst I [22]. Pour la signification de N, A, B, se reporter à la légende de la figure 1. 


\section{REFERENCES}

18. Nishida $Y$, Miki T, Hisajima $H$, Honjo $T$ Cloning of human immunoglobulin $\varepsilon$ chain genes: Evidence for multiple $\mathrm{C} \varepsilon$ genes. Proc Natl Acad Sci USA 1982; 79: 3833-7.

19. Flanagan JG, Rabbitts TH. Arrangement of human immunoglobulin heavy chain constant region genes implies evolutionary duplication of a segment containing $\gamma, \varepsilon$ and $\alpha$ genes. Nature I982; 300: 709-13

20. Battcy J, Max EE, McBride WO, Swan D, Leder P. A processed human immunoglobulin $\varepsilon$ gene has moved to chromosome 9. Proc Natl Acad Sci USA i982; 79: 5956-6o.

21. Lef ranc $G$, Chaabani $H$, van Loghem $E$, Lef ranc MP, de Lange G, Helal AN. Simultaneous absence of the human $\mathrm{IgG}_{1}, \mathrm{IgG}_{2}, \mathrm{IgG}_{4}$ and $\operatorname{Ig} A_{1}$ subclasses: immunological and immunogenetical considerations. Eur 7 Immunol 1983; I3: $240-4$

22. Lef ranc MP, Lefranc G, de Lange $G$ et al. Instability of the human immunoglobulin heavy chain constant region locus indicated by different inherited chromosomal delctions. Mol Biol Med 1983; 1 : 207-17.

23. Shimizu A, Takahashi N, Yaoita Y, Honjo T. Organisation of the constant region gene family of the mouse immunoglobulin heavy chain Cell 1982; 28: 499-506

24. Milstein CP, Deverson EV, Kabbitts TH. The sequence of the human immunoglobulin $\mu-\delta$ intron reveals possible vestigial switch segments. Nucleic Acids Res 1984; 12 : 6523-35.

25. Migone $N$, Oliviero $S$, de Lange $G$ et al Multiple gene deletions within the human immunoglobulin heavy chain cluster. Proc Natl Acad Sci USA 1984; 81: 581 1-5.

26. Chaabani H, Bech-Hansen NT, Cox D. A multigene deletion within the immunoglobulin heavy chain region. $\mathrm{Am} \mathrm{J} \mathrm{Hum}$ Genet 1985; 37: I $64-7$ I.

27 Hammarström L, Lefranc G, Lef ranc MP Persson MAA, Smith CIE. Aberrant pattern of anti-carbohydrate antibodies in immunoglobulin class or subclass deficient donors. In: Hanson LA Söderström T, Oxelius V, eds. Immunoglobulin Subclass Deficiencies. Bascl: Karger AG (in press).

28. Hammarström L, Carbonara A, De Marchi M, Lef ranc G, Lef ranc MP, Smith CIE. Generation of the antibody repertoire in individuals with multiple immunoglobulin heavy chain constant region gene deletions (submitted)

29. Rabbitts TH, Flanagan JG, Lefranc MP Flexibility and change within the human immunoglobulin gene locus. In: Chater KF, Cullis CA, Hopwood A, Johnston AWB, Woolhouse HW, cds. Biological consequences of DNA structure and genome arrangement. LQndon and Canberra: de 80 ans, originaire de Sardaigne a été trouvée, quant à elle, hétérozygote pour cette délétion, et pour un quatrième type de délétion affectant les gènes $\psi \varepsilon \mathrm{I}, \alpha_{\mathrm{I}}, \psi \gamma, \gamma_{2}$ et $\gamma_{4}$ [25] (délétion $I V$, figure 4). Cette délétion a été récemment trouvée à l'état homozygote, mais associée à un haplotype différent, chez une autre personne, tunisienne [26] (voir Tableau I).

\section{Implications immunologiques}

Sur le plan de l'immunité humorale, et ne donnent lieu à aucune affection particulière, même en l'absence d'IgGI, IgG2, $\operatorname{IgG}_{4}$ et $\operatorname{Ig} \mathrm{A}_{\mathrm{I}}\left[\mathrm{I}_{3}\right.$, 22] qui représentent environ $90 \%$ des IgG et des IgA totales dans un sérum normal. Des études réalisées ces déficiences sont bien supportées sur les spécificités anticorps des $\mathrm{IgG}_{3}$, seule sous-classe d'IgG présente dans ce cas là, ont montré que le répertoire était normal contre les épitopes protéiques (spécificités principalement portées par les IgG I dans un sérum " normal ") et contre les épitopes glucidiques tels que l'acide teïchoïque, le dextran et les polysaccharides de la capsule du pneumocoque (spécificités restreintes à la sous-classe IgG2 chez l'adulte " normal ") [27, 28]. Ceci pourrait être dû à un usage, par les gènes $\mathrm{C} \gamma_{3}$ et $\mathrm{C} \alpha_{2}$ restants, du répertoire des gènes codant les régions variables des chaines lourdes $(\mathrm{VH})$ et ce, sans restriction d'isotype. L'absence des IgE [25] n'a apparemment aucun effet néfaste dans le contexte où vit la personne concernée. Par ailleurs, deux personnes homozygotes pour

\begin{tabular}{|c|c|c|c|c|}
\hline \multicolumn{5}{|c|}{$\begin{array}{l}\text { Tableau I } \\
\text { AFFECTANT LES GĖNES } \\
\text { EGION CONSTANTE } \\
\text { ES DES IMMUNOGLOBULINES, } \\
\text { ONNES EN BONNE SANTÉ }\end{array}$} \\
\hline Origine & $\begin{array}{l}\text { Nombre } \\
\text { de cas }\end{array}$ & $\begin{array}{l}\text { Immunoglobulines } \\
\text { absentes }\end{array}$ & $\begin{array}{c}\text { Types } \\
\text { de délétions } \\
\text { (voir fig. } 4 \text { ) }\end{array}$ & Références \\
\hline $\begin{array}{c}\text { Famille Hass } \\
\text { Tunisie }\end{array}$ & (dont TAK3*) & $\begin{array}{l}\lg G 1, \lg G 2 \\
\lg G 4, \lg A 1\end{array}$ & $1 / 1$ & 13 \\
\hline \multirow[t]{2}{*}{$\begin{array}{c}\text { Famille Tou } \\
\text { Tunisie }\end{array}$} & 3 & $\begin{array}{l}\lg G 1, \lg G 2 \\
\lg G 4, \lg A 1\end{array}$ & $1 / 1$ & $13,22 \mathrm{~A}^{* *}$ \\
\hline & 2 & $\lg A 1$ & $1 / 11$ & $22 \mathrm{~B}^{*}$ \\
\hline $\begin{array}{l}\text { Italie } \\
\text { du Sud }\end{array}$ & 1 & $\begin{array}{c}\lg G 2, \lg G 4 \\
\lg A 1, \lg E\end{array}$ & III/III & 25 \\
\hline Sardaigne & 1 & $\begin{array}{c}\operatorname{IgG} 2, \lg G 4 \\
\operatorname{IgA} 1\end{array}$ & III/IV & 25 \\
\hline Tunisie & 1 & $\begin{array}{c}\lg G 2, \lg G 4 \\
\lg A 1\end{array}$ & IV /IV & 26 \\
\hline
\end{tabular}

2 petits-fils de TAK3 (famille Hass) ont été également trouvés homozygotes pour la même délétion (M.P.Lefranc et G.Lefranc, résultats non publiés)

"* $A$ et $B$ correspondent aux résultats des hybridations des figures 1, 2 et 3 . Les différents types de délétions comprennent respectivement les gènes suivants. comme schématisé par ailleurs dans la figure 4.

délétion I: $C \gamma 1, \psi \varepsilon 1, C \alpha 1, \psi \gamma, C \gamma 2, C \gamma 4$

délétion II : $\psi \varepsilon 1, C \alpha 1, \psi \gamma$

délétion III: $C \alpha 1, \psi \gamma, C \gamma 2, C \gamma 4, C \varepsilon$

délétion IV : $\psi \varepsilon 1, C \alpha 1, \psi \gamma, C \gamma 2, C \gamma 4$. 


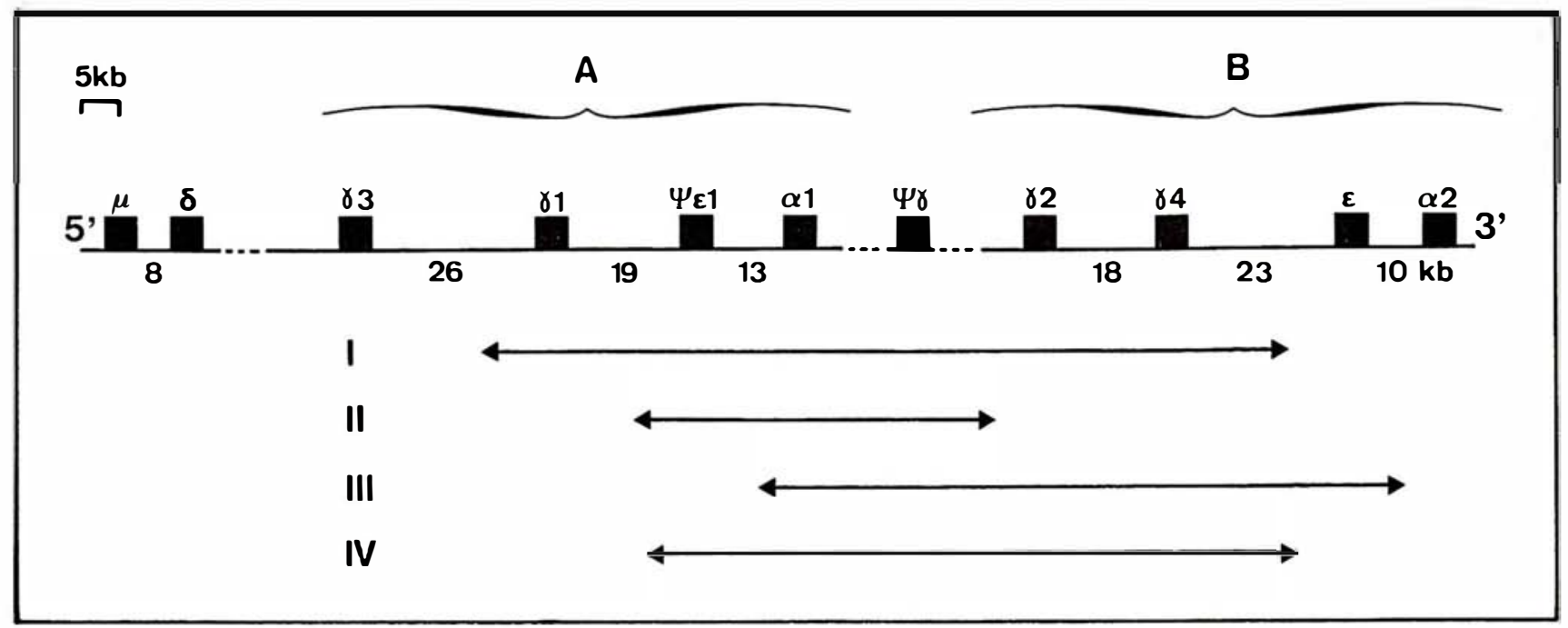

Figure 4. Délétions de gènes de la région constante des chaînes lourdes des immunoglobulines humaines. Ces délétions ont été observées chez des personnes en bonne santé, soit homozygotes pour ces délétions, soit hétérozygotes pour deux délétions différentes (voir Tableau I). Ces délétions I (13, 22), II (22), III (25), N (25, 26) sont décrites dans le texte. L'étendue exacte et le point de jonction de ces différentes délétions restent à déterminer.

la délétion I ont eu cinq grossesses normales donnant naissance à cinq enfants en bonne santé [2 I]. Le taux élevé d'IgG 3 , sous-classe d'IgG la plus facilement transportée à travers le placenta, a permis aux foetus et aux nouveau-nés de survivre jusqu'à ce qu'ils aient synthétisé leurs propres immunoglobulines. Il est probable également que le taux des IgA2 dans le colostrum et le lait maternel ait été plus élevé que la normale permettant ainsi une protection immunologique suffisante dans le tube digestif des jeunes enfants.

\section{Implications \\ immunogénétiques}

La perte d'un ou de plusieurs gènes $\mathrm{CH}$ résulte vraisemblablement soit de recombinaisons inégales au sein de cette famille multigénique ${ }^{*}$, soit de la formation, chez les hétérozygotes, de boucles de délétion. Il est vraisemblable que de telles délé-

* Voir m/s 1985. vol. I, n 4. p. $214-5$.

$m / s n^{\circ} 9$, vol. 2, novembre 86 tions, plus ou moins étendues, affectent avec une fréquence non négligeable $(0,02$ à 0,04$)$, le génome $\mathrm{CH}$ et que le polymorphisme est plus important qu'on ne l'avait imaginé. Mais ces délétions, le plus souvent à l'état hétérozygote, ne peuvent être décelées. Seule la consanguinité élevée régnant dans certaines populations, en augmentant la fréquence des homozygotes pour des haplotypes rares, les rend perceptibles au niveau du phénotype et décelables au niveau moléculaire. Le locus $\mathrm{CH}$ semble posséder une remarquable flexibilité évolutive [29], des délétions et duplications (produits réciproques des recombinaisons inégales) contribuant à modifier le nombre de ses gènes (contraction et expansion). Vraisemblablement les mêmes événements génétiques influent sur la taille des familles de gènes codant pour les régions variables des chaînes légères (VL) et des chaînes lourdes (VH), et par voie de conséquence, sur le répertoire potentiel d'anticorps

\section{Summary}

Extensive multigene deletions have been described in the human immunoglobulin heavychain constant region genes, some of them encompassing more than 100 kilobases. These deletions have all been observed in healthy individuals although these individuals lacked several immunoglobulin subclasses, being either homozygous for one deletion or heterozygous for two different deletions. High frequency of consanguinity in Tunisian population accounts for the increased frequency of individuals displaying one or the other of these deletions in a homozygous state.

\section{TIRES A PART}

M.-P. Lefranc : laboratoire d'immunogénétique, université des sciences et techniques du Languedoc, place E. Bataillon, 34060 Montpellier Cedex. 